\title{
Three-dimensional reconstruction by electron tomography for the application to ultrastructural analysis of SARS-CoV-2 particles
}

\author{
Hong $\mathrm{Wu}^{1} \mathbb{D} \cdot$ Yoshihiko Fujioka $^{1} \cdot$ Shoichi Sakaguchi ${ }^{1} \cdot$ Youichi Suzuki $^{1} \cdot$ Takashi Nakano $^{1}$
}

Received: 17 March 2021 / Accepted: 11 November 2021 / Published online: 26 November 2021

(c) The Author(s) under exclusive licence to The Japanese Society for Clinical Molecular Morphology 2021

\begin{abstract}
SARS-CoV-2 is the cause of COVID-19. The three-dimensional morphology of viral particles existing and multiplying in infected cells has not been established by electron tomography, which is different from cryo-electron tomography using frozen samples. In this study, we establish the morphological structure of SARS-CoV-2 particles by three-dimensional reconstruction of images obtained by electron tomography and transmission electron microscopy of biological samples embedded in epoxy resin. The characteristic roots of spike structures were found to be arranged at the surface of a virion covered with an envelope. A high-electron-density structure that appears to be a nucleocapsid was observed inside the envelope of the virion on three-dimensional images reconstructed by electron tomography. The SARS-CoV-2 particles that budded in the vacuoles in the cytoplasm were morphologically identical to those found outside the cells, suggesting that mature and infectious SARS-CoV-2 particles were already produced in the vacuoles. Here, we show the three-dimensional morphological structure of SARS-CoV-2 particles reconstructed by electron tomography. To control infection, inhibition of viral release from vacuoles would be a new target in the development of prophylactic agents against SARS-CoV-2.
\end{abstract}

Keywords SARS-CoV-2 $\cdot$ Budding $\cdot$ Mature particles $\cdot$ Three-dimensional reconstruction $\cdot$ Transmission electron microscopy $\cdot$ Electron tomography

\section{Introduction}

Severe acute respiratory syndrome corona virus 2 (SARSCoV-2) is the cause of the coronavirus disease 2019 (COVID-19) pandemic [1]. Its outbreak started at Wuhan in Hubei Province, China, in December 2019 [2, 3]. Since then, the world has seen a rapid spread of the virus, quickly

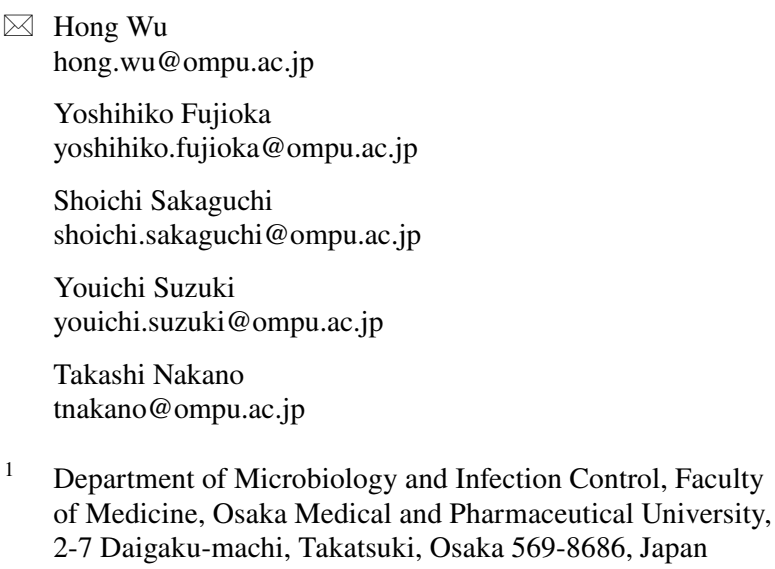

1 Department of Microbiology and Infection Control, Faculty of Medicine, Osaka Medical and Pharmaceutical University, 2-7 Daigaku-machi, Takatsuki, Osaka 569-8686, Japan

infecting an increasing number of people [4]. The transplacental transmission of SARS-CoV-2 remains highly debated [4].

SARS-CoV-2 has been the focus of much study in certain fields such as molecular biology by electron microscopy (EM) [5-7], which is a powerful tool in the field of microbiology. It has played a key role in the rapid identification of viruses in patient samples and has contributed significantly to the clarification of viral structure and function, which help guide the public health response to emerging viral infections [8]. Transmission electron microscopy (TEM) has become a valuable tool to investigate tissues of COVID-19 patients because it allows the visualization of SARS-CoV-2, but the "virus-like particles" found in several organs have been highly contested, because most experts of EM in pathology are not accustomed to analyzing viral particles and subcellular structures [9]. The detailed morphology and ultrastructure of SARS-CoV-2 remains incompletely understood.

In this study, to better understand the three-dimensional (3D) morphological structure of SARS-CoV-2 particles, we attempted to analyze them using conventional TEM and 3D images reconstructed by electron tomography (ET). This is 
a new method of observation and we obtained new information on the viral ultrastructural structure analyzed by ET.

\section{Materials and methods}

\section{Cells and virus}

All cell cultures were maintained at $37{ }^{\circ} \mathrm{C}$ with $5 \% \mathrm{CO}_{2}$. Vero E6/TMPRSS2 cells [10] were obtained from JCRB Cell Bank (Japan) and cultured in Dulbecco's modified Eagle's medium (DMEM, Nacalai Tesque, Inc. Kyoto, Japan) supplemented with $10 \%$ fetal bovine serum (FBS, Life Technologies), 100 units $/ \mathrm{ml}$ penicillin, and $100 \mu \mathrm{g} / \mathrm{ml}$ streptomycin (Nacalai Tesque, Inc., Kyoto, Japan).

The SARS-CoV-2 isolate JPN/TY/WK-521 was kindly provided by the National Institute of Infectious Diseases, Japan. It was propagated in a Vero E6/TMPRSS2 cell culture and stored at $-80{ }^{\circ} \mathrm{C}$ until use. To determine the infectious titer, Vero E6/TMPRSS2 cells were seeded in 24-well plates at $1.5 \times 10^{5}$ cells/well. On the next day, they were incubated with serial dilutions of virus samples in DMEM without FBS at $37{ }^{\circ} \mathrm{C}$ for $2 \mathrm{~h}$. After removal of the supernatant, the cells were cultured in Eagle's minimum essential medium (EMEM, Nissui Pharmaceutical Co., Ltd., Tokyo, Japan) containing $1 \%$ methylcellulose $4000 \mathrm{cp}$ (FUJIFILM, Wako Pure Chemical Corporation, Osaka, Japan) and 2\% FBS. Two days after infection, the cells were fixed with formaldehyde, and plaques were visualized by staining with $1 \%$ crystal violet. The virus titer was calculated as plaque-forming units/ml.

\section{Fixation and preparation of ultrathin sections and staining}

Vero E6/TMPRSS2 $\left(1 \times 10^{6}\right)$ cells were seeded in a $25 \mathrm{~cm}^{2}$ cell culture flask one day prior to infection and exposed to SARS-CoV-2 at a multiplicity of infection (MOI) of 1 for 2 days. Then, the SARS-CoV-2-infected cells were pelleted and fixed with $2 \%$ glutaraldehyde in $0.05 \mathrm{M}$ cacodylate buffer (pH 7.2) at $4{ }^{\circ} \mathrm{C}$ for $3 \mathrm{~h}$. The fixed cells were washed five times with $0.05 \mathrm{M}$ cacodylate buffer and postfixed with $1 \%$ osmium tetroxide in $0.06 \mathrm{M}$ cacodylate buffer for $2 \mathrm{~h}$. The samples were dehydrated in ethanol solutions of serially graded concentrations, embedded in epoxy resin (Nisshin EM, Tokyo, Japan), and then processed using a routine procedure for EM. Ultrathin sections of $100 \mathrm{~nm}$ thickness were prepared for EM using a Reichert-Nissei ultramicrotome (ULTRACUT-N, Nissei Sangyo, Tokyo, Japan) and mounted on a nickel grid (300 mesh) supported by a carbon-coated collodion film. The ultrathin sections were subjected to conventional double staining with $4 \%(\mathrm{w} / \mathrm{v})$ uranyl acetate for 10 min and with Reynolds' $\mathrm{Pb}$ for 3 min for EM.

\section{EM observation and 3D image reconstruction}

All the sections were observed by TEM and ET (H-7800 types, Hitachi, Tokyo, Japan). The accelerating voltage for EM observations was $100.0 \mathrm{kV}$. Electron micrographs were taken with a CCD camera (XR-81) at a magnification of $5000-15,000 \times$, and the pixel size of all micrographs taken by EM with the CCD is 322 ppi and $3296 \times 2472$. Tilted images of a 100-nm-thick ultrathin section were taken using a TEM system equipped with $\mathrm{a} \pm 70^{\circ}$ automated tilting apparatus in which the tilting step angle of sequential images is at $2^{\circ}$ each for ET (HT7800 types, Hitachi, Tokyo, Japan) and the sequential tilted images obtained were reconstructed by the tomography-based reconstruction (TBR) method using 3D reconstruction software (EMIP-EX, Hitachi, Tokyo, Japan). The TBR method enables high-precision ET with few artifacts owing to the suppression of the effect of missing information attributable to the limited sample tilt angle. The detailed process of ET is as follows: electron beam tomography is carried out by computer processing a series of images formed by tilting a specimen consecutively through a specific tilt range. The PC-controlled 3D reconstruction system (HT7800 types, Hitachi, Tokyo, Japan) incorporates a function for automatic image acquisition of a tilted specimen and the software for 3D reconstruction of the acquired tilt series. The automatic tilted-specimen image acquisition function is activated to record TEM images while automatically correcting the position, focus, and magnification of the specimen. On the other hand, EMIP-EX contains a set of programs that have functions for aligning the tilt-axis position (X-and Y-axes and rotation) for each of the consecutively tilted or tilt-series images and also for calculating the data for $3 \mathrm{D}$ reconstruction. The automatic image alignment of a tilt-series image using EMIP-EX is executed in a non-marker-based way. The alignment using the EMIP-EX is executed in combination with the coarse alignment using image correlation method and the fine alignment using correspondence point search method. Fine alignment is executed after extracting the corresponding points automatically from each image. Then, the distribution of 3D shapes and different cross-sectional faces can be displayed according to the results of calculation (Hitachi High-Tech Corporation).

\section{Results}

To obtain 3D reconstructed images of SARS-CoV-2 particles, images were first obtained by conventional EM as shown at Fig. 1. The viral particles containing 
(a)

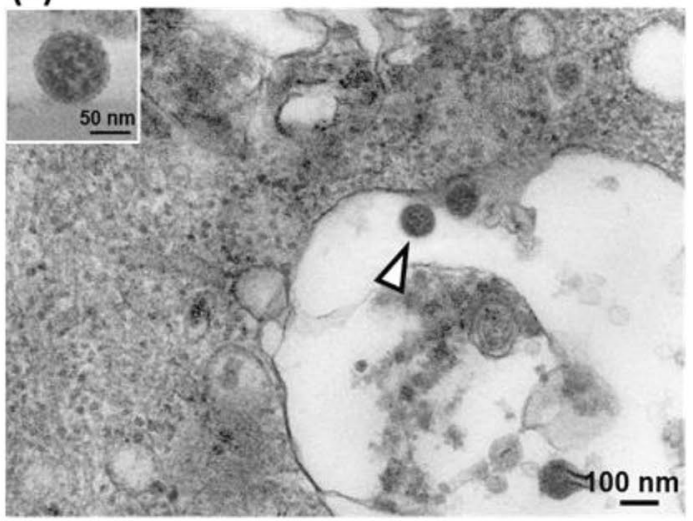

$\left(a^{\prime}\right)$

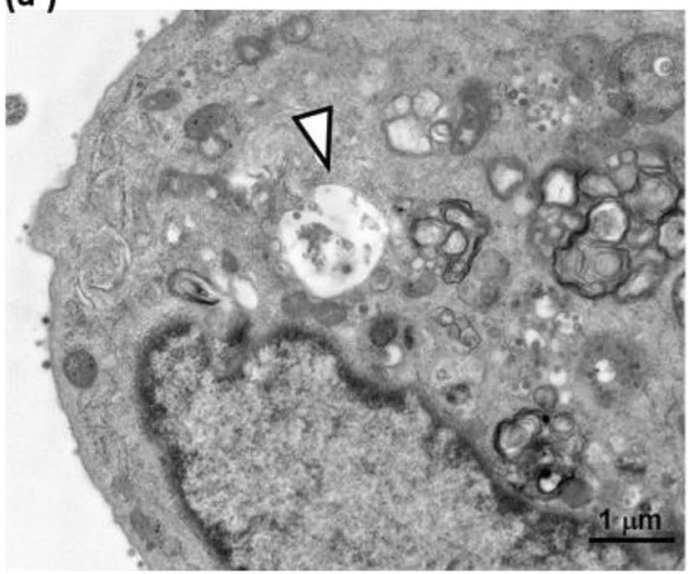

(b)

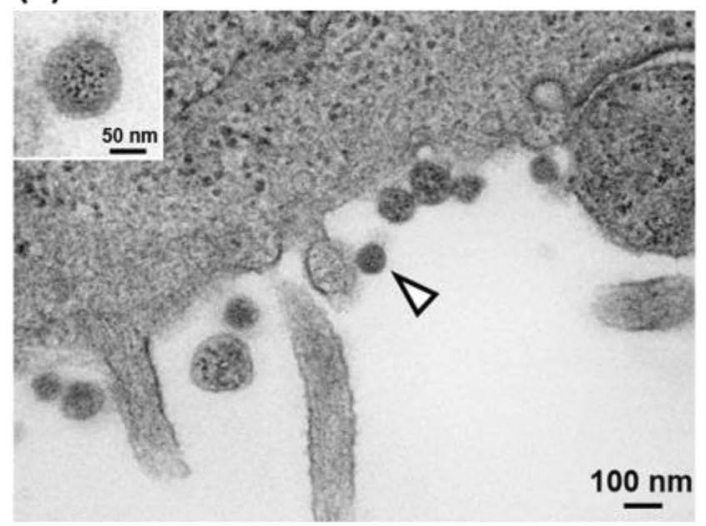

Fig. 1 Conventional EM images of viral particle in SARS-CoV2-infected Vero cell. Conventional TEM of SARS-CoV-2 particle (arrows) in vacuole (a), lower-magnification micrograph of vacuoles (arrows) (a') and virion (arrows) at the surface of a SARS-CoV2-infected Vero cell (b) in ultrathin section. A higher-magnification micrograph of the viral particle is shown in both (a) and (b), as insets. $B a r=100 \mathrm{~nm}$ in both $\mathbf{a}$ and $\mathbf{b}$ images, and $=1 \mu \mathrm{m}$ in $\mathbf{a}^{\prime}$ image

nucleocapsid cores were observed in the images. The diameters of the particles ranged between 100 and $120 \mathrm{~nm}$. Virions containing high-electron-density cores were observed in an ultrathin section. The virions were (a)

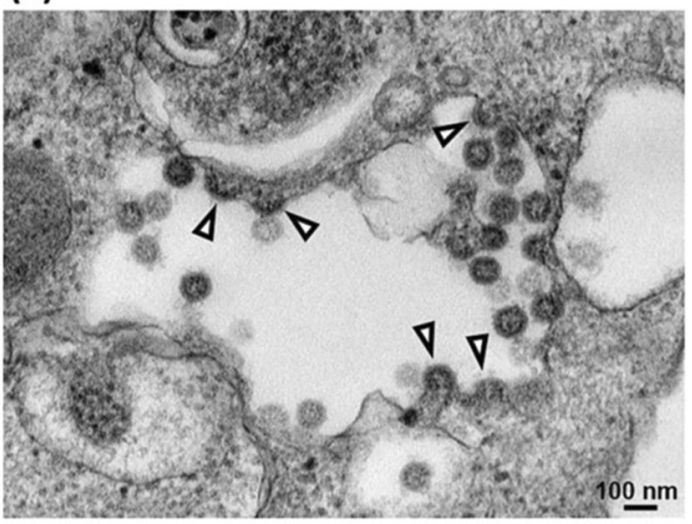

(b)

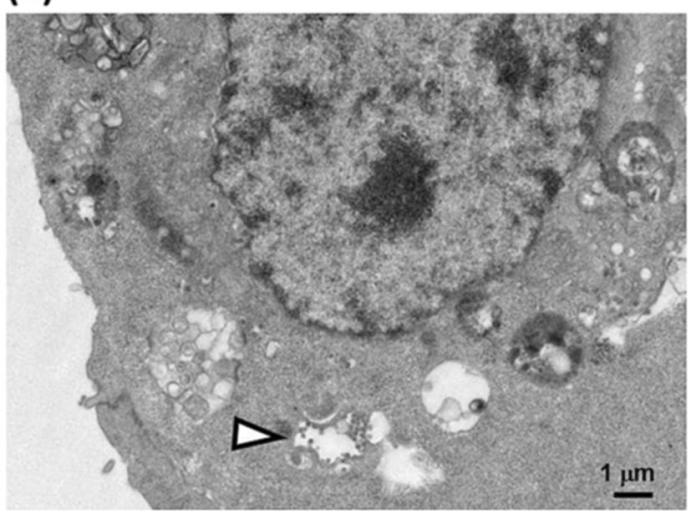

Fig. 2 Conventional EM image of budding viral particle in SARSCoV-2-infected Vero cell. Budding (arrows) of nucleocapsid in membranes of vacuoles (large circular vesicles) containing structural proteins, resulting in circular SARS-CoV-2 particles (a), and lowermagnification micrograph of vacuoles (arrows) (b). Bar $=100 \mathrm{~nm}$ in a image and $=1 \mu \mathrm{m}$ in $\mathbf{b}$ image

observed in vacuoles, as shown in Fig. 1a and a lowermagnification micrograph of vacuoles in Fig. 1a', and at the cell surface (Fig. 1b). The virions appeared to have identical structures. Moreover, the budding of the nucleocapsid in the membranes of vacuoles (large circular vesicles) containing structural proteins was observed as shown in Fig. 2a and a lower-magnification micrograph of vacuoles in Fig. 1b. The budding forms circular viral particles. This finding suggests that the virions in the vacuoles have the same morphology as the viral particles at the cell membrane surface.

To obtain 3D images from the images of a single viral particle shown in Fig. 3a, b, which is the black and white inverse image of Fig. 3a obtained by ET, representative images (3D images in the $\mathrm{Y}$ and $\mathrm{X}$ directions of the Fig. 1a image of the SARS-CoV-2 particle in the vacuole) were obtained. The original video file of the 3D ( $\mathrm{Y}$ and $\mathrm{X}$ directions) images obtained by ET is available as Supplementary Video 1. The characteristic roots of the spikes are arranged 


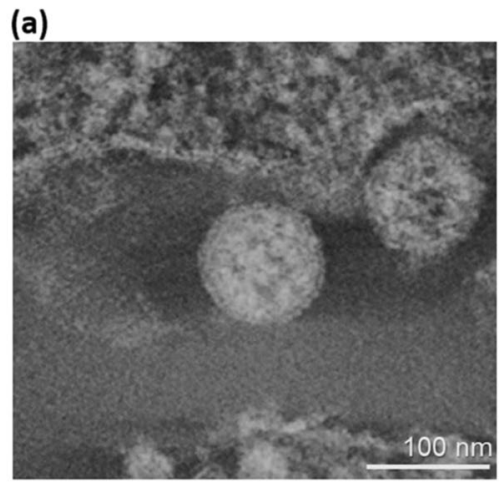

(b)

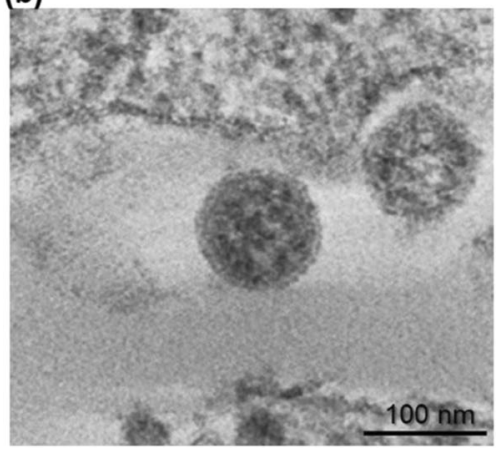

Fig. 3 ET images of virion-like particle in SARS-CoV-2-infected Vero cell (in the vacuole of cell). a ET images of single virus particle of SARS-CoV-2 in the vacuole of SARS-CoV-2-infected Vero cell in ultrathin section and $\mathbf{b}$ inverse image of $\mathbf{a}$. Circles with the solid line indicate virus particles with corona shape or round core, so-called viral particle. Bar $=100 \mathrm{~nm}$ in both images

at the surface of the virion and an envelope covers the particle. A high-electron-density structure that appears to be a nucleocapsid is observed inside the envelope of the virion in 3D sequential ET images. The images of the viral particle obtained from the best shot to identify certain structures are shown in Fig. 4a, b. The roots of the spikes and envelope are observed clearly, as indicated by arrows in the images. The original video file of the $3 \mathrm{D}$ ( $\mathrm{Z}$ slice position) images obtained by ET is available as Supplementary Video 2. A representative 3D image of virions in a vacuole obtained from the best shot to identify certain structures is shown in Fig. 5a. From this slice position, the roots of the spikes are also observed to be located at the surface of the particle, and an envelope covers the particle. A high-electron-density structure that appears to be a nucleocapsid is observed inside the envelope of virion in 3D images obtained by ET. An image of the viral particles obtained from the best shot to identify certain structures is shown in Fig. 5b. The roots of the spikes and envelope are also shown clearly. Figure 5a', $\mathrm{b}^{\prime}$ are the 3D volume rendering of images of the selected areas in Fig. 5a, b, respectively, which show the 3D structure of the nucleocapsid arranged in the particle in 3D images reconstructed by ET (arrows). To present the nucleocapsid,

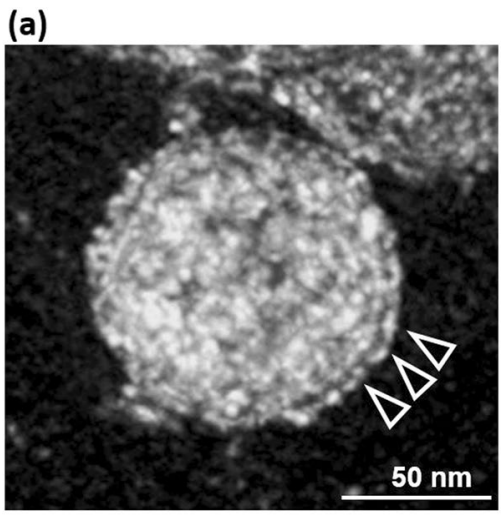

(b)

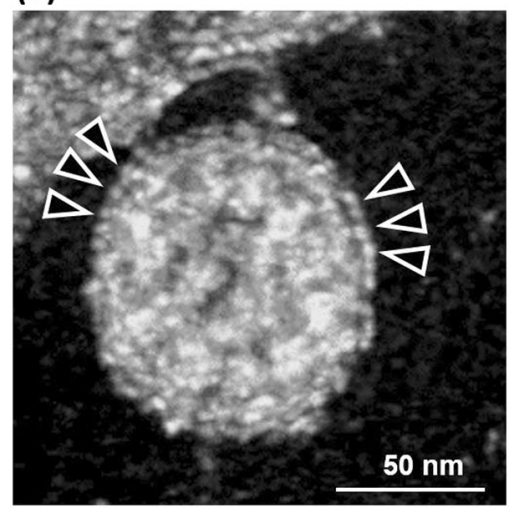

Fig. 4 3D ET images of virion-like particle in $\mathrm{Y}$ and $\mathrm{X}$ rotations in SARS-CoV-2-infected Vero cell. a, b show images of selected areas captured from original Video 1. The roots of the spikes (arrows) are arranged at the surface of the virion and an envelope covers the particle. Bar $=50 \mathrm{~nm}$ in both images

some images at sequential tilting angles (Fig. 5a1-a5, b1-b5) are selected to show the high-electron-density structure that appears to be a nucleocapsid inside the envelope of a virion from 3D sequential ET images (Videos 2 and 3, respectively).

To compare the morphogenesis of the virus particles in vacuoles and on the cell membrane surface and to confirm whether the particles in both locations have the same morphology, the images of a viral particle (arrow) are shown in Fig. 6a, b, which are black and white inverse representative images obtained by ET, representative images (3D images in the $\mathrm{Y}$ and $\mathrm{X}$ directions of the Fig. $1 \mathrm{~b}$ image of the SARSCoV-2 particle at the cell membrane surface) were obtained. The original video file of the 3D images obtained by ET is available as Supplementary Video 3. The roots of the spikes are arranged at the surface of the virion and an envelope covers the particle. A high-electron-density structure that appears to be a nucleocapsid is observed inside the envelope of virion in 3D sequential electron tomography images. An image of the viral particle obtained from the best shot to identify certain structures is shown in Fig. 7. The roots of the spikes and the 
(a)

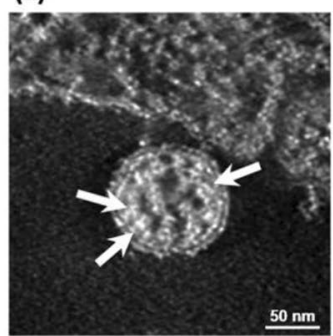

(b)

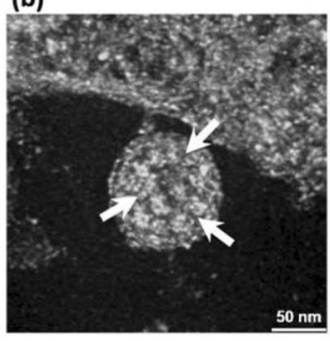

$\left(a^{\prime}\right)$

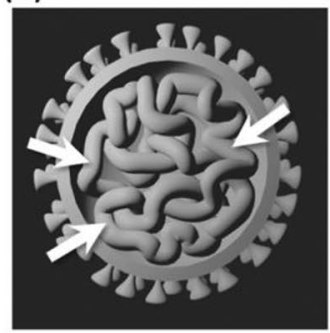

$\left(b^{\prime}\right)$

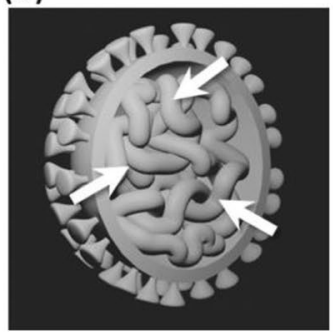

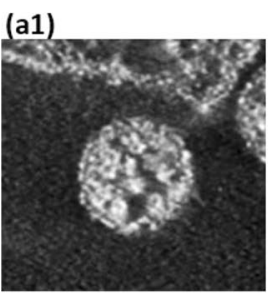

(b1)

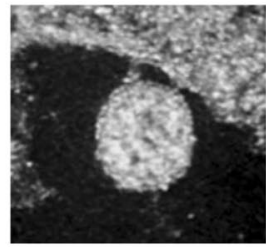

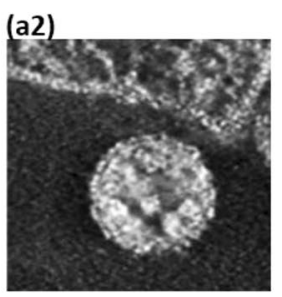

(b2)

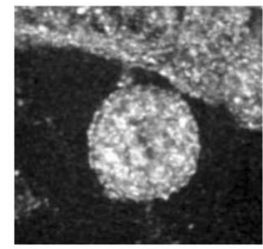

(a3)

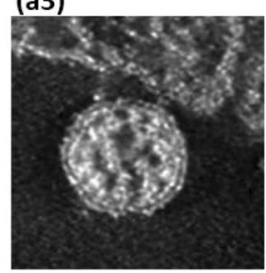

(b3)

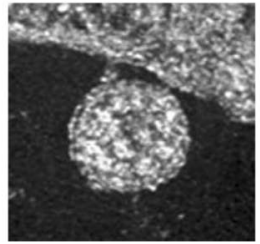

(a4)

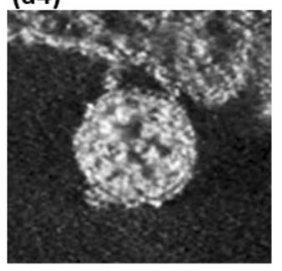

(b4)

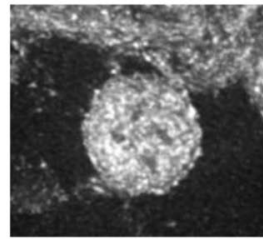

(a5)

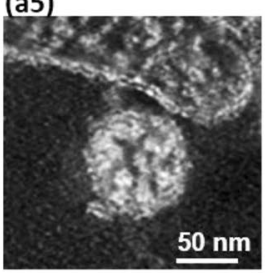

(b5)

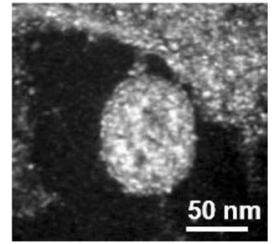

Fig. 5 3D ET images of virion-like particle in SARS-CoV-2-infected Vero cell in $\mathrm{Z}$ slice position. a, b Show images of selected areas captured from original Video 2. The nucleocapsid in the SARS-CoV-2 is shown to have a high-electron-density structure observed inside the envelope of the virion structure in $3 \mathrm{D}$ sequential ET images. $\mathbf{a}^{\prime}, \mathbf{b}^{\prime}$ are the $3 \mathrm{D}$ volume rendering images of the original selected areas in a and $\mathbf{b}$, respectively. The nucleocapsid (arrows) arranged in the particle of 3D images reconstructed by ET. Images at sequential tilting angles (a1-a5 and b1-b5) are selected to show the high-electron-density structure that appears to be a nucleocapsid inside the envelope of the virion from 3D sequential ET images (Videos 2 and 3, respectively). $\mathrm{Bar}=50 \mathrm{~nm}$ in all images envelope are clearly shown (arrows) to be the same as those in the viral particles that were budding in vacuoles. A representative image (3D in $\mathrm{Z}$ slice position) of a viral particle in vacuoles is shown in Supplementary Video 4. The roots of the spikes are observed at the surface of the particle and an envelope covers the particle from this slice position. A highelectron-density structure that appears to be a nucleocapsid is observed inside the envelope of the virion in 3D sequential ET images. An image of the viral particle obtained from the best shot to identify certain structures is shown in Fig. 8. The roots of the spikes and the envelope are also clearly shown in the image. The above findings indicate that the structure of the SARS-CoV-2 particles budding in the vacuoles of the cytoplasm is the same as that found at the cell surface. 


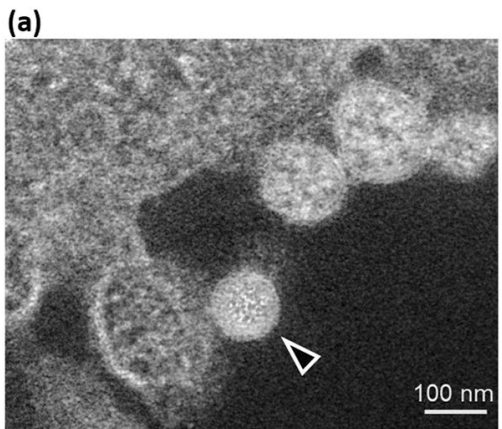

(b)

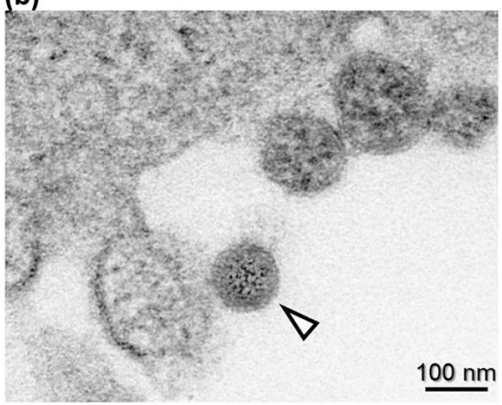

Fig. 6 ET images of virion-like particle in SARS-CoV-2-infected Vero cell (at the cell surface). a ET image of single virus particle (arrows) of SARS-CoV-2 at the cell surface in ultrathin section and b inverse image of a. Circles with the solid line indicate viral particles with corona shape or round core, so-called viral particles. Bar $=100 \mathrm{~nm}$ in both images

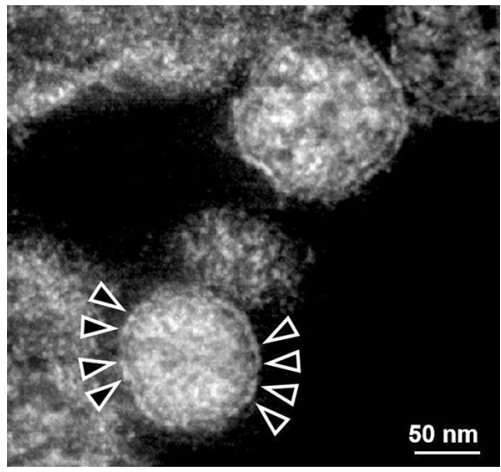

Fig. 7 3D ET image of a viral particle in SARS-CoV-2-infected Vero cell (at the cell surface) in $\mathrm{Y}$ and $\mathrm{X}$ rotations. The image of an area captured from original Video 3 was selected. The roots of the spikes and the envelope are clearly shown (arrows). The nucleocapsid in SARS-CoV-2 is observed to have a high-electron-density structure inside the envelope of the virion structure in $3 \mathrm{D}$ images obtained by sequential ET. Bar $=50 \mathrm{~nm}$

\section{Discussion}

As SARS-CoV-2 continues to spread, it is imperative that we continue to improve our fundamental understanding of its morphology. Morphological analysis of viral particles

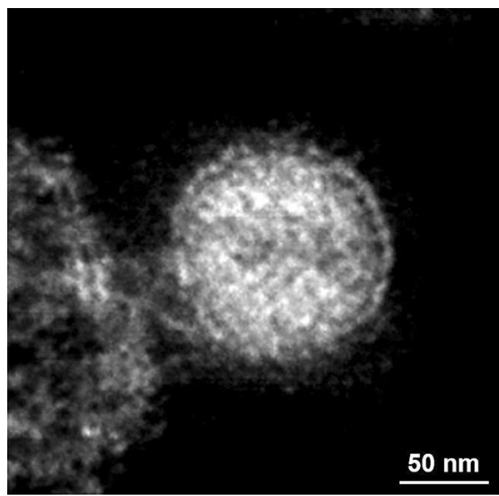

Fig. 8 3D ET image of a viral particle in SARS-CoV-2-infected Vero cell (at the cell surface) in $\mathrm{Z}$ slice position. The image of an area captured from original Video 4 was selected. The nucleocapsid in the SARS-CoV-2 is observed to have a high-electron-density structure inside the envelope of virion in 3D images obtained by sequential ET. $\mathrm{Bar}=50 \mathrm{~nm}$

enables us to diagnose many types of viral disease. In particular, many unknown viral species have been compared with previously determined viral species on the basis of their morphologies. Electron tomographical approaches are based on the imaging of a specimen at different tilt angles by TEM. Recently, ET with an automated tilting apparatus and the TBR algorithm, which follows a similar principle to medical X-ray computed tomography, has been developed and applied for both resin-embedded and frozen samples; such applications are called ET and cryoET, respectively [11]. Technological advances in TEM enable the direct detection on images, automated image collection, and processing algorithms for images providing 2-7-nm-scale axial resolution in the tomographic reconstructions of cells and organelles [11]. Cryo-ET has been applied to the analysis of plunge-frozen SARS-CoV-2 particles $[12,13]$ but it has not been demonstrated whether viral particles budding in vacuoles have the same structure as those on the cell membrane surface. Moreover, one of the advantages of ET is its room-temperature operation, which enables the in situ observation of the behavior of virions in biological tissues or infected cells in 3D images obtained by ET. It is possible to capture 3D images showing how viruses proliferate in the target biological tissue and how they bud in the cells they infected, which may lead to the clarification of the processes involved in virioninfected-cell-to-cell propagation.

Enveloped viral particles assemble in the host cell cytoplasm. In general, viruses are classified into two types according to their virion budding pattern. In one type, newly formed particles emerge and accumulate in vacuoles; subsequently, viral particles accumulated in the vacuoles are released across the cell membrane. Viruses with this type of budding pattern include coronaviruses. In contrast, the other 
type that includes the human immunodeficiency virus (HIV) buds directly from the cell membrane [14]. In this study, we analyzed SARS-CoV-2 particles by conventional TEM with electron tomography. The structure of virions was observed successfully in both 3D images and sliced sequential images. The particles observed by TEM were considered as SARSCoV-2 virions, because they were found only in SARSCoV-2 JPN/TY/WK-521-infected Vero E6/TMPRSS2 cells, but not in uninfected cells (data not shown). In addition, the roots of the spikes were arranged at the surface of the particles, which were covered with an envelope-like structure. Also, a high-electron-density structure that appears to be a nucleocapsid was observed inside the envelope of virion in 3D images obtained by ET. We found that the morphology of SARS-CoV-2 particles in the vacuoles of infected cells is identical to that of particles found outside the cell membrane, suggesting that both particles are mature particles. Moreover, budding was observed only in vacuoles, not on the cell membrane, and immature donut-shaped virus particles were only observed in vacuoles in the cytoplasm, not outside the cell membrane (data not shown). Therefore, we speculated that virion-containing vacuoles fuse with the plasma membrane and finally release viral particles outside the cell. These morphological findings suggest that the virions outside the infected cells are the mature particles released from vacuoles, which may then infect other cells, thus continuing the cell-to-cell propagation. This finding is consistent with previous reports about SARS-CoV-2 budding and release from the vesicles of SARS-CoV-2-infected Vero cells $[15,16]$.

From TEM and ET images, the envelope is present outside the observed viral particle. In addition, a high-electrondensity structure observed inside the envelope of a virion appears to be a nucleocapsid, which is consistent with the characteristics of viral particles, and molecular biological analysis such as PCR analysis also shows similar viral characteristics. Images of the negative control cells are also shown below. We will perform immunoelectron microscopy using an anti-SARS-CoV-2 antibody to detect the virions in our future research.

Our findings also provide new insights into the transmission of SARS-CoV-2. Our detailed method of electron tomography described here in detail may serve as a tool for researchers working on COVID-19 and who are interested in clarifying the complete SARS-CoV-2 infection cycle and the adhesion and infection mechanisms of SARS-CoV-2 virions. Compared with other in situ techniques, TEM and ET are the only methods of visualizing virions found in tissues and will be required to prove SARS-CoV-2 replication outside the respiratory tract.

This is a new report on the reconstruction of 3D images of the morphological structure of SARS-CoV-2 particles by ET applied to plastic-embedded specimens, and it provides new information on the shape and the outline of the structures of the virus and the high-electron-density structure of the nucleocapsid in virions determined by ET. Furthermore, such information obtained by ET of viruses in stored specimens may be helpful for morphological studies such as the re-examination of viruses in huge numbers of pathological samples already pooled.

\section{Conclusion}

We provide the evidence supporting the finding that SARS$\mathrm{CoV}-2$ particles budding in vacuoles in the cytoplasm are morphologically identical to those located outside of cells, suggesting that both types of particle are mature particles. These findings obtained from 3D morphological images may provide new insights into the cell-to-cell propagation and transmission of SARS-CoV-2. To control infection, inhibition of viral release from vacuoles would be a new target in the development of prophylactic agents against SARS-CoV-2.

Supplementary Information The online version contains supplementary material available at https://doi.org/10.1007/s00795-021-00309-2.

Acknowledgements We are grateful to the National Institute of Infectious Diseases (NIID), Japan for providing the SARS-CoV-2 isolate JPN/TY/WK-521. We thank Ms. Akiko Wakui of the Hitachi HighTech Corporation for her technical help in electron tomography. We thank Mr. Taka-aki Ishizuka of the Department of Central Research Laboratory, Osaka Medical and Pharmaceutical University, and Ms. Yukiko Takada of the Department of Microbiology and Infection Control, Osaka Medical and Pharmaceutical University for their technical help. We also thank Ms. Hiromi Norimitsu for her help in the preparation of the manuscript.

\section{References}

1. van Dorp L, Acman M, Richard D, Shaw LP, Ford CE, Ormond L, Owen CJ, Pang J, Tan CCS, Boshier FAT, Ortiz AT, Balloux F (2020) Emergence of genomic diversity and recurrent mutations in SARS-CoV-2. Infect Genet Evol 83:104351

2. Zhu N, Zhang D, Wang W, Li XW, Yang B, Song JD, Zhao X, Huang BY, Shi WF, Lu RJ, Niu PH, Zhan FX, Ma XJ, Wang DY, Xu WB, Wu GZ, Gao GF, Tan WJ (2020) A novel coronavirus from patients with pneumonia in China, 2019. N Engl J Med 382:727-733

3. Wu F, Zhao S, Yu B, Chen YM, Wang W, Song ZG, Hu Y, Tao ZW, Tian JH, Pei YY, Yuan ML, Zhang YL, Dai FH, Liu Y, Wang QM, Zheng JJ, Xu L, Holmes EC, Zhang YZ (2020) A new coronavirus associated with human respiratory disease in China. Nature 579:265-269

4. Caldas LA, Carneiro FA, Higa LM, Monteiro FL, da Silva GP, da Costa LJ, Durigon EL, Tanuri A, de Souza W (2020) Ultrastructural analysis of SARS-CoV-2 interactions with the host cell via high resolution scanning electron microscopy. Sci Rep 10:16099 
5. Park WB, Kwon NJ, Choi SJ, Kang CK, Choe PG, Kim JY, Yun J, Lee GW, Seong MW, Kim NJ, Seo JS, Oh MD (2020) Virus Isolation from the First Patient with SARS-CoV-2 in Korea. J Korean Med Sci 35:e84

6. Pesaresi M, Pirani F, Tagliabracci A, Valsecchi M, Procopio AD, Busardò FP, Graciotti L (2020) SARS-CoV-2 identification in lungs, heart and kidney specimens by transmission and scanning electron microscopy. Eur Rev Med Pharmacol Sci 24:5186-5188

7. Prasad S, Potdar V, Cherian S, Abraham P, Basu A (2020) Transmission electron microscopy imaging of SARSCoV-2. Indian J Med Res 151:241-243

8. Brahim Belhaouari D, Fontanini A, Baudoin JP, Haddad G, Bideau ML, Bou Khalil JY, Raoult D, Scola BL (2020) The strengths of scanning electron microscopy in deciphering SARSCoV-2 infectious cycle. Front Microbiol 11:2014

9. Hopfer H, Herzig MC, Gosert R, Menter T, Hench J, Tzankov A, Hirsch HH, Miller SE (2021) Hunting coronavirus by transmission electron microscopy-a guide to SARS-CoV-2-associated ultrastructural pathology in COVID-19 tissues. Histopathology 78:358-370

10. Matsuyama S, Nao N, Shirato K, Kawase M, Saito S, Takayama I, Nagata N, Sekizuka T, Katoh H, Kato F, Sakata M, Tahara M, Kutsuna S, Ohmagari N, Kuroda M, Suzuki T, Kageyama T, Takeda M (2020) Enhanced isolation of SARS-CoV-2 by TMPRSS2expressing cells. Proc Natl Acad Sci USA 117:7001-7003

11. Otegui MS, Pennington JG (2019) Electron tomography in plant cell biology. Microscopy (Oxford) 68:69-79
12. Klein S, Cortese M, Winter SL, Wachsmuth-Melm M, Neufeldt CJ, Cerikan B, Stanifer ML, Boulant S, Bartenschlager R, Chlanda $\mathrm{P}$ (2020) SARS-CoV-2 structure and replication characterized by in situ cryo-electron tomography. Nat Commun 11:5885

13. Wolff G, Limpens RWAL, Zevenhoven-Dobbe JC, Laugks U, Zheng S, de Jong AWM, Koning RI, Agard DA, Grünewald K, Koster AJ, Snijder EJ, Bárcena M (2020) A molecular pore spans the double membrane of the coronavirus replication organelle. Science 369:1395-1398

14. Garoff H, Hewson R, Opstelten DJ (1998) Virus maturation by budding. Microbiol Mol Biol Rev 62:1171-1190

15. Mandala VS, McKay MJ, Shcherbakov AA, Dregni AJ, Kolocouris A, Hong M (2020) Structure and drug binding of the SARS-CoV-2 envelope protein transmembrane domain in lipid bilayers. Nat Struct Mol Biol 12:1202-1208

16. Xian Y, Zhang J, Bian Z, Zhou H, Zhang Z, Lin Z, Xu H (2020) Bioactive natural compounds against human coronaviruses: a review and perspective. Acta Pharm Sin B 7:1163-1174

Publisher's Note Springer Nature remains neutral with regard to jurisdictional claims in published maps and institutional affiliations. 